\title{
An SC-FDMA Channel Estimation Algorithm Research Based on Pilot Signals
}

\author{
Xue Jianbin, Li Songbai \\ School of Computer and Communication \\ Lanzhou University of Technology \\ Lanzhou, China \\ xue_jabn@hotmail.com, rackby@163.com
}

\begin{abstract}
SC-FDMA as LTE uplink main technology has been widely studied,at the same time, channel estimation around such technology is becoming hot,In order to research and increase channel estimation accuracy of LTE uplink system,an algorithm based on pilot is proposed in this paper,this algorithm's main idea is,by setting a certain bit error rate,choosing some proper data after channel estimation done under R-LS and M-MMSE, combining the chosen data and former block pilot and increasing pilot number to do channel estimation again, system bit error rate and mean square error results can be got by simulation;at last,the theoretical analysis and simulation results show that this algorithm really can make the channel estimation accuracy improved and bit error rate decreased.
\end{abstract}

Keywords-channel estimation; block pilot; bit error rate; SCFDMA;LTE

\section{INTRODUCTION}

OFDMA(Orthogonal Frequency Division Multiple Access) is not suitable for LTE(Long Term Evolution) uplink,because it asks for high power amplifier and transmitting power from transmitting terminal,such requirements can hardly be met for a mobile equipment whose direction is smaller,smarter and long battery life.SCFDMA avoids OFDMA's defects without changing OFDM transmission structure, what's more, it combines traditional technologies of FDMA and single carrier transmission, and can let multiuser share communication resources.that's why SC-FDMA has been chosen as LTE uplink main technology and widely used in wireless communication.

With the development of SC-FDMA,channel estimation around such technology is becoming a research focus.LTE study group has no firm rule on channel estimation methods, but has a rule that its uplink has massive pilot pattern.At present,commonly used pilot channel estimation algorithms include LS(Least Square),MMSE (Minimum Mean Square Error), and channel estimation based on transformation domain,etc.Literature[1] proposed a symbol decision algorithm for fast time-varying channel estimation,but its accuracy is not good enough;literature[2] presented a combination channel estimation algorithm that has good performance when SNR is between $7 \sim 15 \mathrm{~dB}$, but it decreases when SNR is other value;literatures[3-4] had a research on LS, MMSE and decision feedback algorithm,the simulation results show that MMSE has a better performance; TNMSE(Truncated Normalized Mean Squared Error) algorithm proposed in literature[5] overcomes sideband effect, which is reflected by the limited length Fourier series approximation of Gibbs phenomenon, and shows that MMSE channel estimation can withstand minimum sideband recession;R-LS (Regularization-LS) algorithm and M-MMSE(Mismatch-MMSE) algorithm are respectively proposed in literatures[6-7],the feasibility of theoretical analysis is verified shown in its simulation results. The algorithm proposed in this paper is based on block type pilot and combines R-LS and M-MMSE, the simulation results show that higher channel estimation precision can be obtained through the limited time iterative estimation, which means that this algorithm is feasible.

\section{SySTEM ModULE}

SC-FDMA based on OFDMA has a DFT module and an IDFT module respectively in its sender and receiving end.Signals after being modulated and coded are firstly transformed into multipath parallel signals through serialparallel transformation, then go into subcarrier mapping after $N$ points DFT;multipath signals become a serial signal after parallel-serial transformation and $M$ points IFFT, then CP (Cyclic Prefix) is added on the signal,finally,such serial signal is transmitted into wireless channel after DAC(Digital to Analogue Conversion) and RF,receiving end has a nearly opposite process.System block diagram is shown as Figure.1

With normal CP,the basic frame structure of SC-FDMA in time domain is $10 \mathrm{~ms}$ long and equally divided into 20 time slots, each slot is $0.5 \mathrm{~ms}$ long and has 7 SC-FDMA symbols, in frequency domain,each slot has 12 sub-carriers and each subcarrier bandwidth is $15 \mathrm{kHz}$,pilot signal location is shown as Figure 2,block type pilot locates in every slot's forth symbol.At anytime t,the received signals under multipath can be presented as:

$$
y(t)=x(t) * h(t)+w(t) .
$$

here, ${ }^{*}$ is convolution operation, $h(t)$ is channel shock response, $w(t)$ is addictive noise, suppose the modulated signal is $x=\left[x_{1}, x_{2}, x_{\mathrm{N}}\right]$ T, after $N$ points DFT: $X=F_{\mathrm{N}} \bullet x$, here, $F_{\mathrm{N}}$ is DFT matrix whose element is:

$$
(\boldsymbol{F})_{n, m}=\frac{1}{\sqrt{N}} \exp \left(-\frac{j 2 \pi n m}{N}\right) \cdot
$$

here, $n, m \in[0, N-1], N$ is the length of SC-FDMA symbol,the received signal is: 


$$
y=x * h+w
$$

CP turns linear convolution into circular convolution, and FFT changes circular convolution into frequency domain's product,represented as:

$$
\boldsymbol{Y}=\boldsymbol{X H}+\boldsymbol{W} .
$$

here, $\boldsymbol{Y}$ is received signal, $\boldsymbol{X}$ is transmitted signal, $\boldsymbol{H}$ is frequency domain channel response, $\boldsymbol{W}$ is Gaussian white noise. The structure of SC-FDMA pilot is block type pilot that can be produced by $\mathrm{ZC}^{[8-9]}$ serial,a $L_{\mathrm{zc}}$ long $\mathrm{ZC}$ serial $q_{\mathrm{b}}$ is shown as:

$$
q_{b}(s)=\left\{\begin{array}{c}
\exp \left(-j 2 \pi b \frac{s^{2} / 2+s d}{L_{z c}}\right), L_{z c}=2 a \\
\exp \left(-j 2 \pi b \frac{s(s+1) / 2+s d}{L_{z c}}\right), L_{z c}=2 a+1
\end{array} .\right.
$$

here, $a$ is natural number, $b \in\left\{1,2, L_{\mathrm{ZC}}-1\right\}$ is $\mathrm{ZC}$ serial radical exponent, $s=0,1, \cdots, L_{\mathrm{ZC}^{-}}-1, d$ is any integer,for simplicity, suppose $d=0^{[10]}$, then signal with pilot is:

$$
\hat{\boldsymbol{X}}=\left[\hat{X}_{1}, \hat{X}_{2}, \cdots, \hat{X}_{N}\right]^{T} .
$$

after subcarrier mapping:

$$
\overline{\boldsymbol{X}}=\boldsymbol{A} \cdot \hat{\boldsymbol{X}}
$$

here, $\boldsymbol{A}$ is zero padding matrix:

$$
\mathbf{A}=\left[\begin{array}{ccccc}
1 & 0 & \cdots & 0 & 0 \\
& & \mathbf{0}_{v \times N} & & \\
0 & 1 & & 0 & 0 \\
\vdots & \vdots & \mathbf{0}_{v \times N} & \vdots & \vdots \\
0 & 0 & \cdots & 0 & 1
\end{array}\right]_{M \times N} .
$$

here, $v \in\{0,1, \ldots,|M / N|\}$ and $M>N$, There are two subcarrier mapping ways of SC-FDMA: localized FDMA and distributed FDMA,their main difference is zero padding among sub-carriers, when inserting $v$ " 0 " among each DFT sampling output signals, it's localized FDMA if $v=0$, and each user's bandwidth is $1 / U$ of system bandwidth;it's distributed FDMA if $v>0$, each user's bandwidth is expanded as long as system bandwidth;this paper adopts the former. Then, after $M$ points IFFT, we get:

$$
\overline{\boldsymbol{X}}=\boldsymbol{F}_{M}^{H} \cdot \hat{\boldsymbol{X}} .
$$

here, $\boldsymbol{F}_{M}{ }^{H}$ is IFFT matrix,then,multiplying a stack factor $\boldsymbol{C}$ that means inserting $\mathrm{CP}$ to eliminate ISI(Inter Symbol Interference), then signal with $\mathrm{CP}$ is:

$$
\tilde{\boldsymbol{X}}=\boldsymbol{C} \cdot \bar{x} .
$$

$$
\boldsymbol{C}=\left[\begin{array}{cc}
\boldsymbol{0}_{L_{Z C} \times\left(M-L_{Z C}\right)} & \boldsymbol{I}_{L_{Z C}} \\
\boldsymbol{I}_{M} & \boldsymbol{0}
\end{array}\right]_{\left(L_{Z C}+M\right) \times M} .
$$

I is unit matrix,then keep going as Figure 1,SC-FDMA signals are produced and transmitted into wireless channel.

\section{THE AlgORITHM's BASIC IDEA}

After a range of treatments,SC-FDMA symbols arrive at the receiving end,some symbols that have error bits and are under a certain threshold will be extracted and put into channel estimator with pilot for quadratic estimate,if necessary,more estimates can be done till achieving desired results. block digram is:

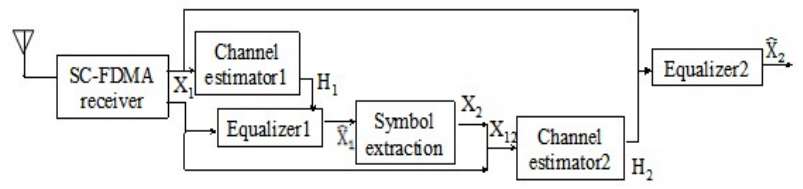

Fig 3. Algorithm block diagram

In Figure.3, channel response $\mathrm{H}_{1}$ comes out from channel estimatorl,we can get the first channel estimated and equalized result $\hat{X}$, by putting H1 and SC-FDMA symbols in equalizerlif channel estimation accuracy or BER meets the requirements, keep moving as Figure 1,if not,set a certain BER,extract some reliable signals from the first estimation result and put it in channel estimator2 with pilot,then, get the secondary channel estimation and equalization results $\hat{X}_{2}$, more iterative computations can be made if necessary, the threshold BER comes from the relationship of BER and $\mathrm{SNR}^{[11]}$ shown as formula(12)-(16) :

$$
B E R=\frac{1}{2}\left[1-\frac{\mu}{\sqrt{2-\mu^{2}}}\right] .
$$

here, $\mu=\mathrm{SNR} /(1+\mathrm{SNR})$, Suppose $\boldsymbol{X}_{\mathrm{n}}$ is transmitted signal,and $\boldsymbol{Y}_{\mathrm{n}}$ is received signal, $\boldsymbol{H}_{\mathrm{n}}$ is channel response in frequency domain, $\boldsymbol{W}_{\mathrm{n}}$ is Gaussian white noise, the signal received on the $n$th subcarrier must be:

$$
\boldsymbol{Y}_{n}=\boldsymbol{X}_{n} \boldsymbol{H}_{n}+\boldsymbol{W}_{n} .
$$

after channel estimation,the signal and SNR are:

$$
\begin{gathered}
\hat{\boldsymbol{X}}_{n}=\boldsymbol{Y}_{n} / \boldsymbol{H}_{n}=\boldsymbol{X}_{n}+\boldsymbol{W}_{n} / \boldsymbol{H}_{n} . \\
S N R_{\hat{\boldsymbol{X}}_{n}}=\sigma_{n}^{2} /\left(\sigma_{N_{n}}^{2} / \boldsymbol{H}_{n}\right)=|\boldsymbol{H}(n)|^{2} S N R_{x} .
\end{gathered}
$$

Obviously,SNR is function of channel grain $H(n)$,if $\mathrm{SNR}_{\text {threshold }}$ is determined, and $|H(n)|^{2} S N R_{x} \geq S N R_{\text {threshold }}$, what means:

$$
|\boldsymbol{H}(n)| \geq \boldsymbol{H}_{\text {threshold }}=\sqrt{S N R_{\text {threshold }} / S N R_{x}} .
$$

then,the threshold $H_{\text {threshold }}$ is determined,so the chosen symbol is proper as long as its grain is higher than this threshold. 


\section{Performance Simulation AND Analysis}

\section{A. Theoretical Analysis}

For LS algorithm,suppose the pilot symbol is $g, \tilde{\boldsymbol{H}}_{g}$ is $g \times 1$ Gaussian white noise vector,and its variance is $\sigma_{\tilde{H}_{g}}^{2}$, then, the channel estimation $\hat{\boldsymbol{H}}_{g}$ is:

$$
\hat{\boldsymbol{H}}_{g}=\boldsymbol{H}_{g}+\tilde{\boldsymbol{H}}_{g}=\boldsymbol{F}_{g} \boldsymbol{h}+\tilde{\boldsymbol{H}}_{g} .
$$

here, $g \in(0, \cdots, G)$ is useful pilot,h is $L \times 1$ channel shock response, $L$ is channel maximum delay length,and $L \leq L_{\mathrm{cp}}, F_{\mathrm{g}}$ is $G \times L$ matrix, $\boldsymbol{F}_{L}$ is $N \times L$ matrix which has the lines where pilot locates and the former columns of $N \times N$ DFT matrix, $\tilde{\boldsymbol{H}}_{G}$ is $G \times 1$ Gaussian white noise vector, then the whole channel shock response is:

$$
\hat{\boldsymbol{H}}_{L S}=\boldsymbol{F}_{L}\left(\boldsymbol{F}_{G}^{H} \boldsymbol{F}_{G}\right)^{-1} \boldsymbol{F}_{G}^{H} \hat{\boldsymbol{H}}_{G} .
$$

as the reference literature [6] shows that parameters chosen in LTE system makes LS algorithm can not be directly applied to SC-FDMA,But classical and robust R-LS algorithm can.Here,expression $\left(\boldsymbol{F}_{G}^{H} \boldsymbol{F}_{G}\right)^{-1}$ is an illconditioned matrix because there are some unmodulated sub-carriers in SC-FDMA system, this problem can be resolved by adding a normalized matrix $\alpha \boldsymbol{I}_{\mathrm{L}}$ to avoid inversing matrix,here $\alpha$ is a constant,usually, it is $0 \sim 1$, the system has best overall performance when $\alpha=1^{[12]}$, so the estimation performance can be improved in given SNR range, channel response in frequency domain is:

$$
\hat{\boldsymbol{H}}_{R-L S}=\boldsymbol{F}_{L}\left(\boldsymbol{F}_{G}^{H} \boldsymbol{F}_{G}+\alpha \boldsymbol{I}_{L}\right)^{-1} \boldsymbol{F}_{G}^{H} \hat{\boldsymbol{H}}_{G} .
$$

its $\mathrm{MSE}(\mathrm{Mean}$ Square Error)is:

$$
M S E_{R-L S}=E\left[\left\|\hat{\boldsymbol{H}}_{R-L S}-\boldsymbol{H}\right\|^{2}\right] .
$$

simplified as:

$$
M S E_{R-L S}=\sigma_{\tilde{\boldsymbol{H}}_{g}}^{2} \boldsymbol{F}_{L}\left(\boldsymbol{F}_{G}^{H} \boldsymbol{F}_{G}+\alpha \boldsymbol{I}_{L}\right)^{-1} \boldsymbol{F}_{G}^{H} .
$$

For M-MMSE algorithm,its MMSE estimation result is:

$$
\hat{\boldsymbol{H}}_{M M S E}=\boldsymbol{F}_{L}\left(\boldsymbol{F}_{G}^{H} \boldsymbol{F}_{G}+\sigma_{\tilde{H}_{G}}^{2} \boldsymbol{C}_{h}^{-1}\right)^{-1} \boldsymbol{F}_{G}^{H} \hat{\boldsymbol{H}}_{G} .
$$

here, $C_{h}=\mathrm{E}\left[h h^{\mathrm{H}}\right], \mathrm{MMSE}$ is statistical estimation, which needs second order statistics(power delay and noise variance).To avoid second order statistical estimation and real-time inversing matrix that is for MMSE direct application, suppose channel power delay spectrum is evenly distributed, then, $C_{\mathrm{h}}$ has identity matrix structure, expression $(27)$ can be improved as:

$$
\hat{\boldsymbol{H}}_{M-M M S E}=\boldsymbol{F}_{L}\left[\boldsymbol{F}_{G}^{H} \boldsymbol{F}_{G}+\left(\sigma_{\tilde{H}_{G}}^{2} / \sigma_{G}^{2}\right) \boldsymbol{I}_{L}\right]^{-1} \boldsymbol{F}_{G}^{H} \hat{\boldsymbol{H}}_{G} \cdot
$$

and,its MSE is:

$$
M S E_{M-M M S E}=E\left[\left\|\hat{\boldsymbol{H}}_{M-M M S E}-\boldsymbol{H}\right\|^{2}\right] .
$$

simplified as:

$$
M S E_{M-M M S E}=\left\|\boldsymbol{R}_{H H}-\boldsymbol{R}_{H H}\left(1+\frac{\beta}{S N R}\left(\boldsymbol{R}_{H H}\right)^{-1}\right)^{-1}\right\| \cdot
$$

here, $R_{H H}=\mathrm{E}\left\{H H^{\mathrm{H}}\right\}$ is autocorrelation matrix of channel response, then, the average SNR is:

$$
S N R=E|\boldsymbol{X}(n)|^{2} / \sigma_{g}^{2} .
$$

and:

$$
\beta=E\left|\boldsymbol{X}_{12}(g)\right|^{2} / E\left|1 / \boldsymbol{X}_{12}(g)\right|^{2} .
$$

here, $\beta$ is a constant that is dependent on modulation, $\beta=1$ when the modulation is QPSK.

\section{B. Simulation Results}

Simulation in this paper is based on LTE uplink SCFDMA, for simplicity, $U=2$, carrier bandwidth is $5 \mathrm{MHz}, \mathrm{FFT}$ sampling points $M=512, L_{\mathrm{cp}}=M / 8$, Doppler frequency shift is $50 \mathrm{~Hz}$, subcarrier number is 300 ,subcarrier mapping is localized FDMA,modulation is QPSK, bit error rate threshold is 0.005 ,twice iterative computations, 1000 times of Monte Carlo simulation on each SNR node,channel is typical city macro cell model,simulation results are shown as Figure 4 and Figure 5.

Figure 4 shows the MSE performance curves of two kinds of estimation methods, under the condition that channel diameter number is known or estimation is accurate and exists error,whether M-MMSE or R-LS, performance of second estimation result is better than that of first estimation, and M-MMSE performance has about 2 3 dB gain than R-LS algorithm,each estimation method has no error basement,estimation performance gains of both have obvious rising trend when SNR growing, but that trend slows down when SNR is higher.

Figure 5 shows system BER curves of two kinds of estimation methods, when SNR is low,estimation accuracy can not be improved because the signals chosen from estimator1 are not enough,but estimation precision rises when the selected symbol number and SNR increasing, obviously, result from estimator2 is better than that from estimator1, and estimator 2 of R-LS is $5 \mathrm{~dB}$ better than estimator1,such two algorithms nearly have error basement when SNR is close to $18 \mathrm{~dB}$,grains of such two algorithms have almost linear change and have no obvious rise when SNR is higher,

\section{CONCLUSION}

Each user in SC-FDMA system occupies only part band and sub-channel has time-frequency related properties, an SC-FDMA channel estimation based on pilot is proposed, from the above theoretical analysis and simulation results, such channel estimation algorithm is feasible in LTE uplink system,because it not only improves channel estimation precision,but also reduces system BER.The only defect is that such algorithm becomes complex when making iterative 
computations, the actual hardware design feasibility and economy efficiency may not be obvious,so,how to improve estimation precision and reduce the algorithm complexity at the same time is still a problem that is worth studying.

\section{ACKNOWLEDGMENT}

This research is financially supported by the National Natural Science Fund Project of China(No.61062002) and the Master Student Tutor Fund Education Department of Gansu Province (No.1014ZTC109).

\section{REFERENCES}

[1] Bao Guan,Xiaojun Wang,Xiaowen Zhang.etc.A new decision feedback detection algorithm of LTE uplink.The Airforce Radars Journal,2010.24(4).

[2] Chaowu Zhan.An OFDM Channel estimation Algorithm Based on Pilot Signals.TV technology.2010,34 (11).

[3] RANA M M.Channel estimation techniques and LTE.terminal implementation challenges //Proc. $13^{\text {th }}$ Inter national Conference on Computer and Information Technology.Dhaka:International Conference Press, 2010,p.545-549.

[4] HUANG G,NIX A,ARMOUR S.Decision feedback equalization in SC-FDMA//Proc.IEEE19 ${ }^{\text {th }}$ International Symposium on Penonal, Indoor ad Mobile Radio Communication. [S.1.]:IEEE Press,2008:1-5.

[5] J.W.Gibbs,'Fourier Series'.Nature,Uol.59,p.200,1898.
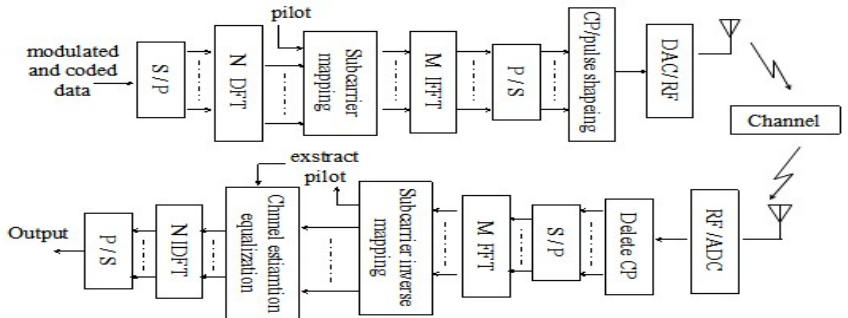

Fig 1. SC-FDMA transmit-receive system(include pilot)

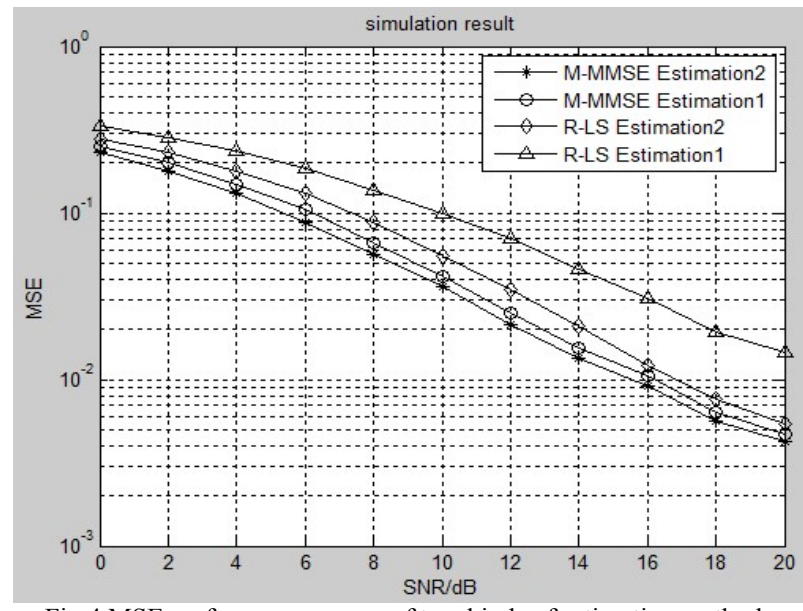

Fig 4.MSE performance curves of two kinds of estimation methods
[6] A.Ancora,C.Bona and Slock D.T.M.,'Down-Sampled Impulse Response Least-Squares Channel Estimation for LTE OFDMA'in Proc.IEEE International Conference on Acoustics, Speech and Signal Processing (ICASSP2007) (Hawaii,USA),2007(4).

[7] P.Hoher,S.Kaiser and P.Robertson,'Pilot--Symbol-aided Channel Estimation Methods in Time and Frequency'in Proc.Communication Theory Mini-Conf.(CTMC)within IEEE Global Telecommun. Conf. (Globecom1997) (Phoenix, USA), 1997 (7)

[8] J.D.C.Chu,'Polyphase Codes with Good Periodic Correlation Properties'.IEEE Trans.on Information Theory, Vol.18, p.531532,1972(7)

[9] R.Frank,S.Zadoff and R.Heimiller,'Phase Shift pulse Codes With Good Periodic Correlation Properties'. IEEE Trans.on Information Theroy, Vol.8,p.381-382,1962(10).

[10] Stefania Sesia,Issam Toufik,Matthew Baker. LTE-The UMTS Long Term Evolution From Theory to Practice[M].Wiley Publishing,Inc. 2011.p.122-163.

[11] J G.PROAKIS,Digital communication. New York: $5^{\text {th }}$ ed. [S.1.]: Mc Graw Hill,2009.

[12] Jan-Japp van de Beek,Ove Edfors,Magnus Sandell, et. On Channel Estimation in OFDM.IEEE.1995.

[13] 3GPP TS $36.211,3^{\text {rd }}$ generation partnership project; technical specification group radic access network; evolved universal terrestrial radio access(E-UTRA); physical channel and modulation (release 9) [S]. 2010

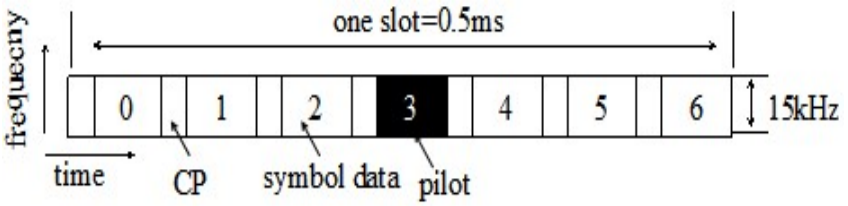

Fig 2. SC-FDMA pilot signal location(include normal CP)

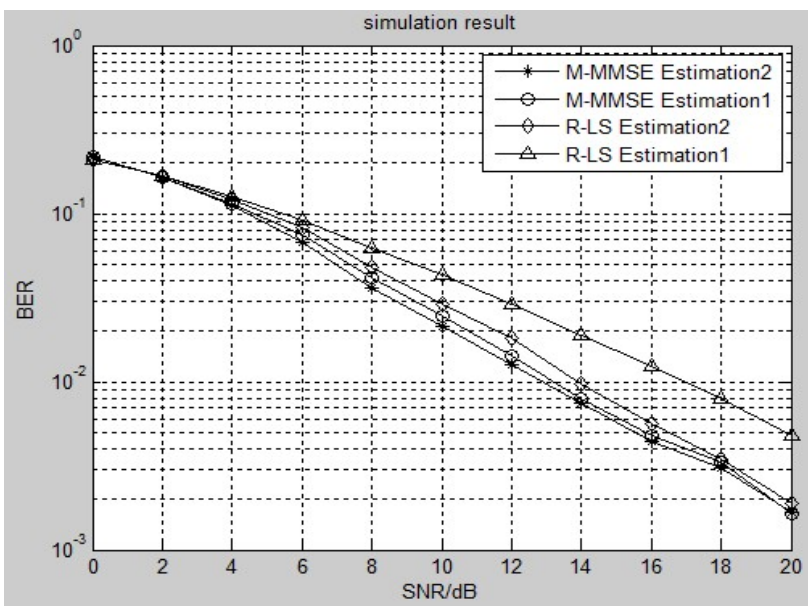

Fig 5.System BER curves of two kinds of estimation methods 